\title{
A NOTE ON BADLY APPROXIMABLE LINEAR FORMS
}

\author{
MUMTAZ HUSSAIN
}

(Received 8 June 2010)

\begin{abstract}
In this paper we investigate the analogue of the classical badly approximable setup in which the distance to the nearest integer $\|\cdot\|$ is replaced by the sup norm $|\cdot|$. In the case of one linear form we prove that the hybrid badly approximable set is of full Hausdorff dimension.
\end{abstract}

2010 Mathematics subject classification: primary 11J25; secondary $11 \mathrm{~J} 13$.

Keywords and phrases: Diophantine approximation, systems of linear forms, Hausdorff dimension.

\section{Introduction}

Let $X=\left(x_{i j}\right) \in \mathbb{I}^{m n}:=(0,1]^{m n}$ be an $m \times n$ matrix. Let

$$
q_{1} x_{1 i}+q_{2} x_{2 i}+\cdots+q_{m} x_{m i} \quad(1 \leq i \leq n)
$$

be a system of $n$ linear forms in $m$ variables. The system will be written more concisely as $\mathbf{q} X$. The classical result of Dirichlet [3] states that for any point $X \in \mathbb{I}^{m n}$, there exist infinitely many integer points $\mathbf{q} \in \mathbb{Z}^{m}$ such that

$$
\|\mathbf{q} X\|:=\max _{1 \leq i \leq n}\left\|q_{1} x_{1 i}+q_{2} x_{2 i}+\cdots+q_{m} x_{m i}\right\|<|\mathbf{q}|^{-m / n},
$$

where $|\mathbf{q}|$ denotes the supremum norm; that is, $|\mathbf{q}|:=\max \left\{\left|q_{1}\right|,\left|q_{2}\right|, \ldots,\left|q_{m}\right|\right\}$, and $\|\cdot\|$ denotes the distance to the nearest integer in $\mathbb{Z}^{n}$. The right-hand side of (1.1) may be sharpened by a constant $c(m, n)$ but the best permissible values for $c(m, n)$ are unknown except for $m=n=1$. A point $X \in \mathbb{I}^{m n}$ is said to be badly approximable if the right-hand side of (1.1) cannot be improved by an arbitrary positive constant. Denote the set of all such points as $\operatorname{Bad}(m, n)$; that is, $X \in \operatorname{Bad}(m, n)$ if there exists a constant $C(X)>0$ such that

$$
\|\mathbf{q} X\|>C(X)|\mathbf{q}|^{-m / n} \quad \forall \mathbf{q} \in \mathbb{Z}^{m} \backslash\{\mathbf{0}\} .
$$

The set $\operatorname{Bad}(1,1)$ is the standard set of badly approximable numbers and corresponds to those irrationals with bounded continued fraction expansion. A consequence of a fundamental theorem of Khintchine in the theory of Diophantine

Research supported by Higher Education Commission of Pakistan: 3-6-25/HEC/Overseas/2006/822.

(C) 2011 Australian Mathematical Publishing Association Inc. 0004-9727/2011 \$16.00 
approximation is that $\operatorname{Bad}(1,1)$ is of zero Lebesgue measure. Nevertheless, a classical result of Jarník [7] states that $\operatorname{Bad}(1,1)$ is a large set in the sense that it has maximal dimension. More precisely, $\operatorname{dim} \operatorname{Bad}(1,1)=1$ where $\operatorname{dim} A$ denotes the Hausdorff dimension of the set $A$-see [5] for the definition of Hausdorff dimension. In higher dimensions, the Khintchine-Groshev theorem [8] implies that $\operatorname{Bad}(m, n)$ is of $m n$-dimensional Lebesgue measure zero and a result of Schmidt [9] states that $\operatorname{dim} \operatorname{Bad}(m, n)=m n$.

In this note we investigate the hybrid of $\operatorname{Bad}(m, n)$ in which the distance to the nearest integer $\|\cdot\|$ is replaced by the sup norm $|\cdot|$. The corresponding wellapproximable theory has been well developed over the years and the hybrid wellapproximable sets naturally appear in operator theory and KAM theory-see [2, 4].

A consequence of the Dirichlet type theorem established by Dickinson in [1] is the following statement.

LEMMA (Dickinson). For each $X \in \mathbb{I}^{m n}$ there exist infinitely many nonzero integer vectors $\mathbf{q} \in \mathbb{Z}^{m}$ such that

$$
|\mathbf{q} X|<m|\mathbf{q}|^{-m / n+1} .
$$

In view of this lemma, it is natural to consider the following badly approximable set. Let $\mathbf{B a d}^{*}(m, n)$ denote the set of $X \in \mathbb{I}^{m n}$ for which there exists a constant $C(X)>0$ such that

$$
|\mathbf{q} X|>C(X)|\mathbf{q}|^{-m / n+1} \quad \forall \mathbf{q} \in \mathbb{Z}^{m} \backslash\{\mathbf{0}\} .
$$

REMARK 1.1. In the case where $m=n$, it is easily seen that

$$
\mathbb{I}^{m^{2}} \backslash\left\{X \in \mathbb{I}^{m^{2}}: \operatorname{det} X=0\right\}=\operatorname{Bad}^{*}(m, m) .
$$

Now

$$
\left|\left\{X \in \mathbb{I}^{m^{2}}: \operatorname{det} X=0\right\}\right|_{m^{2}}=0
$$

where $|\cdot|_{k}$ denotes $k$-dimensional Lebesgue measure. Hence, it follows that

$$
\left.\operatorname{Bad}^{*}(m, m)\right|_{m^{2}}=1
$$

and so $\operatorname{dim} \operatorname{Bad}^{*}(m, m)=m^{2}$. The upshot of this is that in the case $m=n$ the corresponding badly approximable set is of full dimension.

In the case where $m>n$, the Khintchine-Groshev type theorem recently established in [6] implies that the $m n$-dimensional Lebesgue measure of $\operatorname{Bad}^{*}(m, n)$ is zero; that is,

$$
\left|\operatorname{Bad}^{*}(m, n)\right|_{m n}=0 .
$$

Naturally, one would expect that the following analogue of Schmidt's theorem is true.

Conjecture. For $m>n, \operatorname{dim} \operatorname{Bad}^{*}(m, n)=m n$.

In this note we establish the conjecture for one linear form $(n=1)$ in $m$ variables.

THEOREM 1.2. For $m \geq 1$,

$$
\operatorname{dim} \mathbf{B a d}^{*}(m, 1)=m
$$




\section{Proof of Theorem 1.2}

In view of the above remark, we can assume without loss of generality that $m \geq 2$. Next, since $\operatorname{Bad}^{*}(m, 1) \subseteq \mathbb{I}^{m}$, we immediately have that

$$
\operatorname{dim} \operatorname{Bad}^{*}(m, 1) \leq m \text {. }
$$

Thus the proof of Theorem 1.2 follows on obtaining the complementary lower bound estimate. For this we shall make use of the following result.

LEMMA 2.1. Let $S$ be a subset of $\mathbb{I}^{k}$ and let

$$
\Lambda:=\{(x, x S): x \in \mathbb{I}\} .
$$

If $\operatorname{dim} S=k$, then $\operatorname{dim} \Lambda=k+1$.

Proof OF LEMma 2.1 Trivially, $\Lambda \subseteq \mathbb{I}^{k+1}$ and so it follows that $\operatorname{dim} \Lambda \leq k+1$. For the reverse inequality, suppose that $\operatorname{dim} \Lambda=h<k+1$. Then given any $\epsilon>0$ and $\delta>0$ such that $h<k+1-\delta<k+1$, there exists a covering $\mathcal{C}$ of $\Lambda$ by $(k+1)$ dimensional hypercubes such that

$$
\sum_{C \in \mathcal{C}}|C|^{k+1-\delta}<\epsilon .
$$

For any fixed $x \in \mathbb{I}$ the set $A:=\{x\} \times x S$ can be covered by the collection

$$
\mathcal{C}(x):=\left\{\left(\{x\} \times \mathbb{I}^{k}\right) \cap C: C \in \mathcal{C}\right\}
$$

of $k$-dimensional hypercubes. Let

$$
\lambda_{C}(x)= \begin{cases}1 & \text { if }\left(\{x\} \times \mathbb{I}^{k}\right) \cap C \neq \emptyset \\ 0 & \text { if }\left(\{x\} \times \mathbb{I}^{k}\right) \cap C=\emptyset,\end{cases}
$$

so that

$$
\int_{0}^{1} \lambda_{C}(x) d x=|C|
$$

and

$$
\sum_{C \in \mathcal{C}(x)}|C|^{k-\delta}=\sum_{C \in \mathcal{C}} \lambda_{C}(x)|C|^{k-\delta} .
$$

Then

$$
\begin{aligned}
\int_{0}^{1} \sum_{C \in \mathcal{C}(x)}|C|^{k-\delta} d x & =\sum_{C \in \mathcal{C}}\left(\int_{0}^{1} \lambda_{C}(x) d x\right)|C|^{k-\delta} \\
& =\sum_{C \in \mathcal{C}}|C|^{k+1-\delta} \\
& <\epsilon .
\end{aligned}
$$

Hence $\mathcal{C}(x)$ is a cover for $A$ such that

$$
\sum_{C \in \mathcal{C}(x)}|C|^{k-\delta}<\epsilon .
$$


In particular, $\mathcal{C}(1)$ is a cover for $S$ and thus $\operatorname{dim} S \leq k-\delta<k$. This contradicts our hypothesis that $\operatorname{dim} S=k$. Hence, $h \geq k+1$ as required.

With reference to Lemma 2.1, with $k=m-1$ and $S:=\operatorname{Bad}(m-1,1)$, we show that $\Lambda$ is contained in the set $\operatorname{Bad}^{*}(m, 1)$.

For each $\mathbf{x} \in \operatorname{Bad}(m-1,1)$ there exists some constant $c(\mathbf{x})>0$ such that

$$
\left|q_{1}+q_{2} x_{2}+\cdots+q_{m} x_{m}\right|>c(\mathbf{x})\left|\mathbf{q}^{*}\right|^{-(m-1)} \quad \forall\left(q_{1}, \mathbf{q} *\right) \in \mathbb{Z}^{m} \backslash\{\mathbf{0}\}
$$

where $\mathbf{q} *:=\left(q_{2}, \ldots, q_{m}\right)$. Multiplying by $x_{1} \in \mathbb{I}$, we get that

$$
\left|q_{1} x_{1}+q_{2} x_{1} x_{2}+\cdots+q_{m} x_{1} x_{m}\right|>x_{1} c(\mathbf{x})\left|\mathbf{q}^{*}\right|^{-(m-1)} \quad \forall\left(q_{1}, \mathbf{q} *\right) \in \mathbb{Z}^{m} \backslash\{\mathbf{0}\} .
$$

Now let $\mathbf{q}:=\left(q_{1}, \ldots, q_{m}\right)$. Then $|\mathbf{q}|=\left|\mathbf{q}^{*}\right|$ if $\left|q_{1}\right| \leq\left|\mathbf{q}^{*}\right|$ and $\left|\mathbf{q}^{*}\right|^{-(m-1)}>$ $|\mathbf{q}|^{-(m-1)}$ if $\left|q_{1}\right|>\left|\mathbf{q}^{*}\right|$. This, together with (2.1), implies that

$$
\left|q_{1} x_{1}+q_{2} x_{1} x_{2}+\cdots+q_{m} x_{1} x_{m}\right|>c\left(\mathbf{x}, x_{1}\right)|\mathbf{q}|^{-(m-1)} \quad \forall \mathbf{q} \in \mathbb{Z}^{m} \backslash\{\mathbf{0}\},
$$

where $c\left(\mathbf{x}, x_{1}\right):=x_{1} c(\mathbf{x})>0$. The upshot of this is that

$$
\Lambda \subseteq \operatorname{Bad}^{*}(m, 1)
$$

Lemma 2.1 implies that $\operatorname{dim} \operatorname{Bad}^{*}(m, 1) \geq m$ and thereby completes the proof of Theorem 1.2.

\section{A final comment}

In the case where $m<n$, although the right-hand side of the inequality appearing in (1.2) is an increasing function as $|\mathbf{q}| \rightarrow \infty$, we cannot rule out the possibility that $|\mathbf{q} X|$ grows faster than $O\left(|\mathbf{q}|^{-m / n+1}\right)$ for some $X$. However, we suspect that this is rare and it is reasonable to expect that

$$
\operatorname{dim} \operatorname{Bad}^{*}(m, n)=0 .
$$

We hope to pursue this and the conjecture in the near future.

\section{Acknowledgements}

I am grateful to my supervisor Sanju Velani for suggesting this problem, and for enthusiastic help and encouragement. I would like to thank the referee for a careful reading of the paper.

\section{References}

[1] H. Dickinson, 'The Hausdorff dimension of the systems of the simultaneously small linear forms', Mathematika 40 (1993), 367-374.

[2] D. Dickinson, T. Gramchev and M. Yoshino, 'Perturbations of vector fields on tori: resonant normal forms and diophantine phenomena', Proc. Edinb. Math. Soc. 45 (2002), 731-759.

[3] L. Dirichlet, 'Verallgemeinerung eines Satzes aus der Lehre von den Kettenbrüchen nebst einigen Anwendungen auf die Theorie der Zahlen', S.-B. Preuss. Akad. Wiss. (1842), 93-95. 
[4] M. M. Dodson and J. A. G. Vickers, 'Exceptional sets in Kolmogorov-Arnol'd-Moser theory', J. Phys. A 19 (1986), 349-374.

[5] K. J. Falconer, Fractal Geometry—Mathematical Foundations and Applications (Wiley, New York, 1990).

[6] M. Hussain and J. Levesley, 'The metrical theory of simultaneously small linear forms', Preprint, arXiv:0910.3428.

[7] V. Jarník, 'Über die simultanen Diophantischen Approximationen', Math. Z. 33 (1931), 505-543.

[8] A. Khintchine, 'Einige Sätze über Kettenbrüche, mit Anwendungen auf die Theorie der Diophantischen Approximationen', Math. Ann. 92 (1924), 115-125.

[9] W. M. Schmidt, 'Badly approximable systems of linear forms', J. Number Theory 1 (1969), 139-154.

MUMTAZ HUSSAIN, Department of Mathematics and Statistics,

La Trobe University, Melbourne, Victoria 3086, Australia

e-mail:m.hussain@latrobe.edu.au 